\title{
Cognitive Implications in Epilepsy.
}

Osama abdelraouf Morad, Mohammed Gama Abdellatif, Ahmed Gamal Arafa, Ahmed Ezzat Amin

\author{
Neurology and Psychological Medicine department, Faculty of Medicine, \\ Sohag University
}

\begin{abstract}
Cognitive disability is a major contributor to the comorbidities of epilepsy. There is major evidence that confirms that cognitive impairment can appear or worsen with early and chronic progressive neurologic changes in epilepsy. It has been increasingly accepted that comorbidity does not indicate causality. Certainly, cognitive impairment in epileptic patients warrants crucial evaluation and mitigation from the onset of diagnosis and treatment of epilepsy. The concept of a bidirectional nature of cognitive impairment in epilepsy represents a change in the paradigm of the neuropsychology of epilepsy. It has been suggested that both behavioral and cognitive dysfunction associated with epilepsy are not necessarily the consequence of active epilepsy but in fact, can dominate and be associated with factors before the emergence of epilepsy. This review discusses different relations of behavioral and cognitive comorbidities in epilepsy and tries to clarify the nature of the relationship between epilepsy and cognition.
\end{abstract}

Keywords: Cognition, epilepsy, comorbidities, behavior

\section{INTRODUCTION}

Epilepsy is defined as recurrent transient attacks of excessive synchronous neuronal activity [1]. Clinical epilepsy includes various manifestations ranging from minimal clinical symptoms like brief unnoticed loss of consciousness to violent attacks of muscular contractions leading to physical injury. Epileptic seizures can be transient caused by brain traumas, drug-induced, hypoxia and metabolic derangement disturbing brain homeostasis [2].

Although epilepsy is a very common disease affecting nearly sixty-five million patients worldwide [3], there is still no obvious concepts for the mechanisms underlying the variable spectrum of manifestations [4].

Cognitive functions are higher brain functions, determined by the ability of the brain to plan adaptive behavior, memorize events, find a solution to
Cognitive dysfunctions, mood disorders, and behavioral problems constitute highly common comorbidities of epilepsy [6].

The most frequent cognitive deficits secondary to epilepsy are memory loss, cognitive slowing, and deficits in attention which have a significant negative effect on daily functions [5].

Cognitive impairment in chronic epilepsy is noticed in $70-80 \%$ of patients as well as depression in $60 \%$ of patients [7].

It is well known that the causal relation of cognitive dysfunction in epilepsy has multiple factors including organic brain lesions, uncontrolled epilepsy, drug treatment, and previous individual mental abilities [8]. Although postictal and ictal cognitive impairment is reversible [9], chronic and uncontrolled epilepsy has commonly progressive cognitive decline [10]. 
It has been suggested that refractory epilepsy is the main cause of the cognitive problems seen with epilepsy. However, this concept questioned by that comorbidities determine epilepsy outcomes of [11], comorbidities may precede the epilepsy onset; and cognitive deficits accompanying epilepsy may be an expression of a common underlying pathologic process [8].

The causal relation between epilepsy and cognitive impairment was challenged in many studies revealed that cognitive and behavioral comorbidities may come before the onset of epilepsy [12,13].

\section{Determinants of cognitive function in epilepsy}

Many factors affect the cognitive functions in epilepsy including the etiology of epilepsy (especially if there is underlying structural damage), epilepsy syndrome, epilepsy duration, location of the epileptic focus, age of onset, seizure type, seizure frequency, occurrence of status epilepticus, type of anti-epileptic drug and drug dose [5].

\section{Seizure etiology}

In epilepsy induced by cortical dysplasia, the degree of the malformation is a major indicator of cognitive impairment. As well as, organic brain damage like sclerosis of the hippocampus in temporal lobe epilepsy produces marked cognitive disability that may be represented as refractory epilepsy [14].

\section{Type of seizure}

Childhood Absence epilepsy has less cognitive dysfunction than tonic-clonic seizures. However, generalized epilepsies are had higher cognitive deficits than focal epilepsies; and the greatest hazard in status epilepticus patients [15].

\section{Type of epileptic syndrome}

Idiopathic generalized epilepsy is characterized by widespread seizures and has bilateral, synchronous and symmetrical spikes and waves on an electroencephalograph (EEG) [16]. Neuropsychologic researches revealed executive function compromise involving the formation of concepts, reasoning, mental speed, cognitive planning. 18FDG positron emission tomography (PET) reveals low prefrontal cortex glucose metabolism during mental functions [16].

Landau-Kleffner syndrome occurs in early childhood and proves the effect of epileptic discharges on cognitive abilities [5].

The more exposure to abnormal neuronal activity leads to disturbance in learning, language development. Speech reception is impaired early, then an expression of speech declines. These deficits show permanent destruction in the verbal-auditory system [16].

\section{Location of the epileptogenic focus}

Focal temporal lobe epilepsy (TLE) is well known to have specific memory deficits: verbal memory dysfunction in the left lobe and visual memory impairment in the right lobe [8].

Uncontrolled (TLE) is associated with progressive cognitive decline due to the cumulative neurodegenerative effect of neurochemical substances, abnormal cerebral electric discharges and metabolic derangement that exhaust the compensatory brain functions over the years of epilepsy [17].

Transient epileptic amnesia is a TLE subtype presents with amnesia and associated with interictal increased forgetting and autobiographical amnesia [18].

This may be attributed to many causes such as abnormal electrical discharge during sleep that may corrupt memory consolidation which occurs in sleep and organic destruction to the temporal lobe which promotes seizures and disrupts memory processing [19]. Frontal lobe epilepsy causes executive 
dysfunction in the form of disabilities in motor tasks, working memory, attention, planning and mental speed [20]

\section{Seizure control}

Uncontrolled recurrent seizures are linked to increased cognitive deficits. A negative correlation between seizures frequency and neuropsychologic tests results [14]

\section{Age of epilepsy appearance}

Age of beginning of epilepsy is the main factor of the cognitive and behavioral effects of epilepsy. The earlier epilepsy onset in children, the lower their IQ results. The onset of epilepsy before the age of five years is a major risk factor for cognitive decline [5].

\section{Comorbid anxiety and depression}

Epileptic patients have a higher risk (4-5 times) developing depression, suicide, and anxiety than healthy people. This may be attributed to the earlier age of onset, longer duration of epilepsy, refractory seizure, increased and interictal epileptiform activity [20]. Anxiety disorders are associated with attacks of amnesia [21]. Depression has neuropsychological impairments involving processing speed, memory, psychomotor speed, attention, and, and executive tasks [22]. So, the cognitive impacts of depression exaggerate and accelerate any cognitive impairment induced by epilepsy itself.

\section{Anti-seizure drugs}

Anti-epileptic drugs reduce neuronal excitability, which may have a negative effect on cognitive function [23]. Cognitive side effects are notable in patients receiving anti-epileptic polytherapy [24]. Drugs acting on sodium channels have much less cognitive drawbacks. However, the risk is higher in drugs enhancing GABA which may lead to mental slowing and impairments of language production [25]. Drugs acting on presynaptic protein SV2A showed improvement in reaction times and memory [26].

\section{Etiologies of cognitive dysfunction in epilepsy}

Cognitive comorbidities in epilepsy have multiple causes and factors that define the course of cognitive functions such as structural pathologies, seizure types, interictal epileptiform activity, drugs, psychiatric problems, and individual mental reserve capacities [27].

Behavioral comorbidities such as depression can be attributed to a) Reactive depression due to problems related to being epileptic; b) Antiepileptic drug-induced depression; c) Disruption of neural networks handling emotions [28].

Besides the multifactorial concept of behavioral and cognitive comorbidities in epilepsy, it is fundamental to appraise the useful role of some antiepileptic drugs on cognitive functions, mood, seizures control [29] and neurodevelopmental prospects such as neuronal plasticity and cognitive capacities [30].

\section{The influence of chronic epilepsy on cognitive abilities}

The concept that epilepsy harms the neuronal circuits and leads to ongoing cognitive decline is confirmed by cross-sectional studies that studied the relationship between cognitive functions and epilepsy duration [31]. The cognitive morbidities in a chronic epileptic patient can be due to negative chronic effect of recurrent seizures or an initial impairment interacting with the aging process [32].

Determinants of continuous cognitive deterioration in long-standing epilepsies are uncontrolled epilepsy and generalized tonic-clonic type epilepsy, brain trauma, underlying structural epilepsy, and a poor baseline 
cognitive performance. These are prognostic markers for more severe epilepsy and a more progressive cognitive decline in epileptic patients [33, 34].

The impact of epileptic encephalopathies on cognition is beyond underlying epilepsy, as there is relentless progressive cognitive and intellectual deterioration [35]. Patients with status epilepticus suffer secondary harm due to brain anoxia or traumatic injuries which may cause irreversible cognitive deterioration. Neuronal cytotoxicity due to over excitation in status epilepticus may contribute to permanent neuronal damage [36].

Currently, no clear evidence that interictal epileptiform discharge and recurrence of seizures alone can contribute significant cognitive dysfunction or permanent cognitive damage in chronic epileptic patients. More likely it depends on the time before and around epilepsy onset considering the baseline cognitive affection [37]. So, it is necessary to assess cognitive abilities at the beginning of epilepsy to realize the time at which deficits happen and how they affect the disease course.

\section{Cognition in early epilepsy}

Cognitive and behavioral comorbidities in epileptic children are present at the onset of epilepsy and cognition course relies on the underlying pathology, epilepsy dynamics, and seizure control [38].

Risk factors for the presence and severity of cognitive comorbidity in new-onset epilepsies include lack of education, the underlying cause of epilepsy, and generalized tonic-clonic seizure type. Accordingly, cognitive screening should become routinely done for pediatric and adult populations [39]. Assessment of the patient's cognitive functions early at epilepsy onset and before the start of treatment helps to monitor the disease and cognition course [40]. Cognitive deficits are commonly existing when epilepsy starts, and their progression depends on the etiology, treatment effects (positive or negative) and treatment success.

\section{Cognitive impairment as a risk factor epilepsy}

Structural or developmental brain lesions, subclinical epileptiform activity, and behavioral/mental deficits can negatively affect cognition before the onset of epilepsy [41]. The concept that cognitive dysfunction is already present at epilepsy onset does not solve the debate that whether a cognitive disability is a determinant for epilepsy development. Cognitive dysfunction and epilepsy express manifestations of abnormal brain activity. Accordingly, seizure recurrence is markedly associated with brain pathology which also contributes to cognitive deficits [42].

It has been reported that better cognitive performance is an indicator of treatment success [43, 44]. Cognitive impairment is accelerated by epileptiform activity [45]. The epileptic seizure not only relies on the focal activity of the epileptic location but also on the activity of away healthy brain regions [46].

Accordingly, if these away non-focal brain regions are under cognitive activation can block seizure evolution, because epilepsy cannot recruit brain regions that are already functionally occupied. Behavioral seizure control techniques depend on that concept [47].

\section{Enhance Cognitive Outcomes in Epilepsy}

A long-term study of patients after temporal lobe epilepsy surgery revealed that cognitive course is usually stable and may improve if seizures are controlled and antiepileptic drugs can be reduced [48]. Another research studied the effect of a ketogenic diet, which has been applied 
to control seizures in some patients with refractory epilepsy, found that the intervention has beneficial cognitive effects. Although the proposed cognitive benefit could be attributed to seizure control, it appeared independent of the reduction of antiepileptic drugs. The same study found improvements in aspects of attention alertness, and thorough cognitive functions [49].

Many methods have been investigated for enhancement of cognitive and behavioral outcomes in epilepsy including pharmacotherapy and neuromodulatory interventions. It has been reported that there is potential cognition benefit of transcranial direct current stimulation and transcranial magnetic stimulation and of 3 pharmacologic classes: N-methyl-Daspartate noncompetitive antagonist memantine, acetylcholinesterase inhibitors donepezil and galantamine, and the psychostimulant methylphenidate [50]. Research into noninvasive neuromodulator mechanisms is in progress and it has a role in cognitive strengthening through a reduction in epileptic discharges frequency, memory reinforcement during sleep [50]. Concentration on epilepsy only may stop the consideration for any underlying disease pathology [that], when managed, may recover the patient's condition, including cognitive dysfunctions [50].

Finally, there may be a common underlying brain substrate that predisposes epileptic patients to express some behavioral problems, cognitive dysfunctions, or actually the seizures themselves.

Surely there is a bidirectional relation between cognitive comorbidity and epilepsy. Epilepsy can have a temporary and reversible negative effect on cognitive function and interictal epileptiform activities have a negative impact on cognitive abilities.
However, there are cognitive functions can suppress a seizure, depending on behavioral seizure control mechanisms. Epilepsy and cognitive disabilities may share common underlying brain disease. It is highly essential to assess and early screen cognitive functions to determine whether cognitive and behavioral comorbidities induced by epilepsy or its drug treatments or whether cognitive disabilities are consistent with early or late acquired brain pathology.

\section{Conclusion}

The goal of this review to highlight the relations and common causes of cognitive comorbidities in epilepsy and the effect of them on the disease course. We focused on the causal relation between epilepsy and cognitive disability and the impacts of cognition on seizures and epileptic activity. We concluded that there is no current reliable evidence that longstanding epilepsy alone, drives progressive deterioration and irreversible decline of cognitive abilities. Additional occupational therapy, training, education, and psychotherapy is advised for patients with cognitive and behavioral problems. Further future research is highly needed to assess and enhance cognition in epilepsy that focuses on targets other than the processes underlying seizures.

\section{References}

1.Wang, B., et al., Vicus: Exploiting local structures to improve network-based analysis of biological data. PLoS computational biology, 2017. 13(10): p. e1005621.

2.Goldberg, E.M. and D.A. Coulter, Mechanisms of epileptogenesis: a convergence on neural circuit dysfunction. Nature Reviews Neuroscience, 2013. 14(5): p. 337.

3.Ngugi, A.K., et al., Estimation of the burden of active and life-time epilepsy: 
a meta-analytic approach. Epilepsia, 2010. 51(5): p. 883-890.

4.Vezzani, A., Epilepsy and Inflammation in the Brain: Overview and Pathophysiology: Epilepsy and Inflammation in the Brain. Epilepsy currents, 2014. 14(2_suppl): p. 3-7.

5.Lodhi, S. and N. Agrawal, Neurocognitive problems in epilepsy. Advances in psychiatric treatment, 2012. 18(3): p. 232-240.

6.Helmstaedter, C. and J.-A. Witt, Clinical neuropsychology in epilepsy: theoretical and practical issues, in Handbook of clinical neurology. 2012, Elsevier. p. 437-459.

7.Anthony, J.C., W.W. Eaton, and A.S. Henderson, Looking to the future in psychiatric epidemiology. Epidemiologic reviews, 1995. 17(1): p. 240-242.

8.Elger, C.E., C. Helmstaedter, and M. Kurthen, Chronic epilepsy and cognition. The Lancet Neurology, 2004. 3(11): p. 663-672.

9.Helmstaedter, C., C. Elger, and M. Lendt, Postictal courses of cognitive deficits in focal epilepsies. Epilepsia, 1994. 35(5): p. 1073-1078.

10.Keezer, M.R. and J.W. Sander, Comorbidity as an epidemiological construct. The Lancet Neurology, 2016. 15(1): p. 32.

11.Keezer, M.R., S.M. Sisodiya, and J.W. Sander, Comorbidities of epilepsy: current concepts and future perspectives. The Lancet Neurology, 2016. 15(1): p. 106-115.

12.Hesdorffer, D.C., et al., Depression and suicide attempt as risk factors for incident unprovoked seizures. Annals of neurology, 2006. 59(1): p. 35-41.

13.Hesdorffer, D.C., et al., Co-occurrence of major depression or suicide attempt with migraine with aura and risk for unprovoked seizure. Epilepsy research, 2007. 75(2-3): p. 220-223.

14.Aldenkamp, A. and N. Bodde, Behaviour, cognition and epilepsy. Acta Neurologica Scandinavica, 2005. 112: p. 19-25.

15.Lezak, M.D., et al., Neuropsychological assessment. 2004: Oxford University Press, USA.
16.Hommet, C., et al., Idiopathic epileptic syndromes and cognition. Neurosci Biobehav Rev, 2006. 30(1): p. 85-96.

17.Mameniskiene, R., et al., The decay of memory between delayed and longterm recall in patients with temporal lobe epilepsy. Epilepsy \& Behavior, 2006. 8(1): p. 278-288.

18.Butler, C., et al., Transient epileptic amnesia: regional brain atrophy and its relationship to memory deficits. Brain, 2008. 132(2): p. 357-368.

19.Butler, C.R., et al., The syndrome of transient epileptic amnesia. Annals of Neurology: Official Journal of the American Neurological Association and the Child Neurology Society, 2007. 61(6): p. 587-598.

20.McCagh, J., J.E. Fisk, and G.A. Baker, Epilepsy, psychosocial and cognitive functioning. Epilepsy research, 2009. 86(1): p. 1-14.

21.Airaksinen, E., M. Larsson, and Y. Forsell, Neuropsychological functions in anxiety disorders in populationbased samples: evidence of episodic memory dysfunction. Journal of psychiatric research, 2005. 39(2): p. 207-214.

22.Gualtieri, C.T., L.G. Johnson, and K.B. Benedict, Neurocognition in depression: patients on and off medication versus healthy comparison subjects. The Journal of neuropsychiatry and clinical neurosciences, 2006. 18(2): p. 217-225.

23.Aldenkamp, A.P., Effects of antiepileptic drugs on cognition. Epilepsia, 2001. 42: p. 46-49.

24.Brodie, M., et al., Psychomotor impairment and anticonvulsant therapy in adult epileptic patients. European journal of clinical pharmacology, 1987. 31(6): p. 655-660.

25.Aldenkamp, A.P. and G. Baker, $A$ Systematic Review of the Effects of Lamotrigine on Cognitive Function and Quality of Life. Epilepsy Behav, 2001. 2(2): p. 85-91.

26.Lagae, L., Cognitive side effects of antiepileptic drugs. The relevance in childhood epilepsy. Seizure, 2006. 15(4): p. 235-41.

27.Elger, C.E., C. Helmstaedter, and M. Kurthen, Chronic epilepsy and 
cognition. Lancet Neurol, 2004. 3(11): p. 663-72.

28.Hoppe, C. and C.E. Elger, Depression in epilepsy: a critical review from a clinical perspective. Nat Rev Neurol, 2011. 7(8): p. 462-72.

29.Helmstaedter, C. and J.A. Witt, Cognitive outcome of antiepileptic treatment with levetiracetam versus carbamazepine monotherapy: a noninterventional surveillance trial. Epilepsy Behav, 2010. 18(1-2): p. 7480.

30.Helmstaedter, C.A., Prediction of memory reserve capacity. Adv Neurol, 1999. 81: p. 271-9.

31.Jokeit, H. and A. Ebner, Effects of chronic epilepsy on intellectual functions. Prog Brain Res, 2002. 135: p. 455-63.

32.Kaaden, S. and C. Helmstaedter, Age at onset of epilepsy as a determinant of intellectual impairment in temporal lobe epilepsy. Epilepsy Behav, 2009. 15(2): p. 213-7.

33.Hermann, B.P., et al., Cognitive prognosis in chronic temporal lobe epilepsy. Ann Neurol, 2006. 60(1): p. 80-7.

34.Helmstaedter, C., et al., Chronic epilepsy and cognition: a longitudinal study in temporal lobe epilepsy. Ann Neurol, 2003. 54(4): p. 425-32.

35.Avanzini, G., et al., Do seizures and epileptic activity worsen epilepsy and deteriorate cognitive function? Epilepsia, 2013. 54 Suppl 8: p. 14-21.

36.Fujikawa, D.G., Prolonged seizures and cellular injury: understanding the connection. Epilepsy Behav, 2005. 7 Suppl 3: p. S3-11.

37.Helmstaedter, C. and J.A. Witt, Epilepsy and cognition - A bidirectional relationship? Seizure, 2017. 49: p. 83-89.

38.Hermann, B.P., et al., Starting at the beginning: the neuropsychological status of children with new-onset epilepsies. Epileptic Disord, 2012. 14(1): p. 12-21.

39.Wilson, S.J., et al., Indications and expectations for neuropsychological assessment in routine epilepsy care: report of the ILAE Neuropsychology Task Force, Diagnostic Methods
Commission, 2013-2017. Epilepsia, 2015. 56(5): p. 674-681.

40.Helmstaedter, C., et al., Disentangling the relationship between epilepsy and its behavioral comorbidities-the need for prospective studies in new-onset epilepsies. Epilepsy \& Behavior, 2014. 31: p. 43-47.

41.Witt, J.-A. and C. Helmstaedter, Cognition in the early stages of adult epilepsy. Seizure, 2015. 26: p. 65-68.

42.Helmstaedter, C. and J.-A. Witt, Epilepsy and cognition-a bidirectional relationship? Seizure, 2017. 49: p. 8389.

43.Yu, H.-Y., et al., Preoperative IQ predicts seizure outcomes after anterior temporal lobectomy. Seizure, 2009. 18(9): p. 639-643.

44.Malmgren, K., et al., Seizure outcome after resective epilepsy surgery in patients with low IQ. Brain, 2007. 131(2): p. 535-542.

45.Nirkko, A.C., et al., Virtual car accidents of epilepsy patients, interictal epileptic activity, and medication. Epilepsia, 2016. 57(5): p. 832-840.

46.Lehnertz, K., et al., Predictability of uncontrollable multifocal seizurestowards new treatment options. Scientific reports, 2016. 6: p. 24584.

47.Tan, G., et al., Meta-analysis of EEG biofeedback in treating epilepsy. Clinical EEG and neuroscience, 2009. 40(3): p. 173-179.

48.Helmstaedter, C., C. Elger, and V. Vogt, Cognitive outcomes more than 5 years after temporal lobe epilepsy surgery: Remarkable functional recovery when seizures are controlled. Seizure, 2018. 62: p. 116-123.

49.van Berkel, A.A., D.M. IJff, and J.M. Verkuyl, Cognitive benefits of the ketogenic diet in patients with epilepsy: a systematic overview. Epilepsy \& Behavior, 2018. 87: p. 69-77.

50.Jacobs, C.S., K.C. Willment, and R.A. Sarkis, Mini-Review: Noninvasive Cognitive Enhancement in Epilepsy. Frontiers in neurology, 2019. 10: p. 167. 